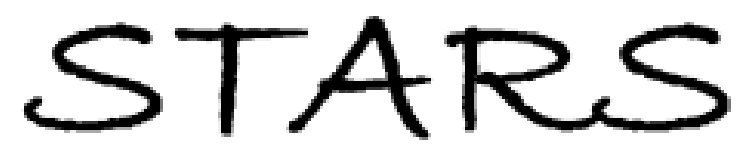

University of Central Florida

STARS

$1-1-2012$

\title{
Modeling of phase volume diffractive gratings, part 2: reflecting sinusoidal uniform gratings, Bragg mirrors
}

Igor V. Ciapurin

Derrek R. Drachenberg

University of Central Florida

Vadim I. Smirnov

George B. Venus

University of Central Florida

Leonid B. Glebov

University of Central Florida

Find similar works at: https://stars.library.ucf.edu/facultybib2010

University of Central Florida Libraries http://library.ucf.edu

This Article is brought to you for free and open access by the Faculty Bibliography at STARS. It has been accepted for inclusion in Faculty Bibliography 2010 s by an authorized administrator of STARS. For more information, please contactSTARS@ucf.edu.

\section{Recommended Citation}

Ciapurin, Igor V.; Drachenberg, Derrek R.; Smirnov, Vadim I.; Venus, George B.; and Glebov, Leonid B., "Modeling of phase volume diffractive gratings, part 2: reflecting sinusoidal uniform gratings, Bragg mirrors" (2012). Faculty Bibliography 2010s. 2425.

https://stars.library.ucf.edu/facultybib2010/2425

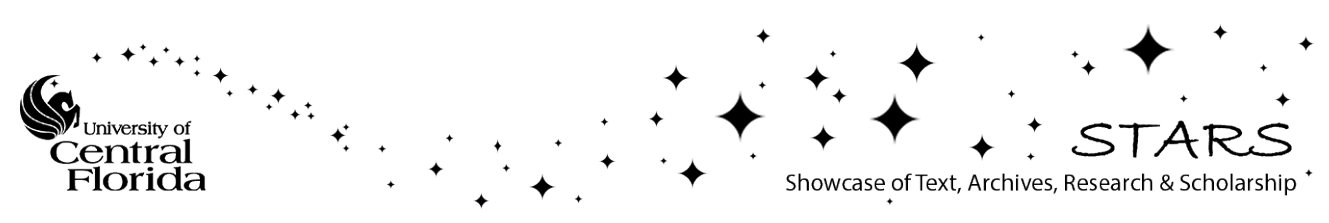




\section{Optical Engineering}

\section{Modeling of phase volume diffractive gratings, part 2: reflecting sinusoidal uniform gratings, Bragg mirrors}

Igor V. Ciapurin

Derrek R. Drachenberg

Vadim I. Smirnov

George B. Venus

Leonid B. Glebov 


\section{Modeling of phase volume diffractive gratings, part 2: reflecting sinusoidal uniform gratings, Bragg mirrors}

\author{
Igor V. Ciapurin \\ OptiGrate Corporation \\ 3267 Progress Drive \\ Orlando, Florida 32826 \\ Derrek R. Drachenberg \\ Lawrence Livermore National Laboratories \\ NIF and Photon Sciences Directorate \\ P.O. Box 808 \\ Livermore, California 94551-0808 \\ and \\ University of Central Florida \\ CREOL/College of Optics and Photonics \\ P.O. Box 162700 \\ Orlando, Florida 32816-2700 \\ E-mail: derrekdrach@gmail.com \\ Vadim I. Smirnov \\ OptiGrate Corporation \\ 3267 Progress Drive \\ Orlando, Florida 32826 \\ George B. Venus \\ Leonid B. Glebov \\ University of Central Florida \\ CREOL/College of Optics and Photonics \\ P.O. Box 162700 \\ Orlando, Florida 32816-2700
}

\begin{abstract}
A detailed model of diffraction of plane and Gaussian beams on plane uniform phase Bragg gratings based on a Kogelnik's theory of coupled waves is presented. The model describes reflecting gratings (Bragg mirrors) with arbitrary orientation in a plane-parallel plate having no material losses. It takes into account spectral width and angular divergence of laser beams. The results of modeling are compared with experimental data for Bragg mirrors in a photo-thermo-refractive glass. ( 2012 Society of Photo-Optical Instrumentation Engineers (SPIE). [DOI: 10.1117/1.OE.51.5.058001]
\end{abstract}

\begin{abstract}
Subject terms: volume Bragg gratings; diffractive optical elements; volume holography; optical design; diffractive optics; Bragg mirrors; retroreflectors; holography applications.
\end{abstract}

Paper 111391 received Nov. 7, 2011; revised manuscript received Feb. 21, 2012 accepted for publication Mar. 29, 2012; published online May 2, 2012; corrected May 8, 2012.

\section{Introduction}

A detailed model of diffraction of plane and Gaussian beams on plane uniform phase Bragg gratings based on a Kogelnik's theory of coupled waves is presented. The model describes reflecting gratings (Bragg mirrors) with arbitrary orientation in a plane-parallel plate having no material losses. It takes into account spectral width and angular divergence of laser beams. The results of modeling are compared with experimental data for Bragg mirrors in a photo-thermo-refractive (PTR) glass.

Wide applications of volume Bragg gratings (VBG) in optics and photonics require comprehensive practical knowledge of such optical elements. In spite of Kogelnik's theory of coupled waves ${ }^{\mathbb{\square}}$ developed about 40 years ago, no reduction to practical formulae enabling precise design of VBGs has been provided up to today. VBGs in the case of a monochromatic plane wave were also treated in detail in Ref. 2, which considers Fresnel reflections and the added effects of composite VBGs such as using two VBGs to form a FabryPerot cavity, but also does not address a reduction to practical formulae for designers.

A detailed modeling of the diffraction of plane monochromatic waves along with divergent and polychromatic beams

0091-3286/2012/\$25.00 @ 2012 SPIE on transmitting volume gratings on the basis of Kogelnik's theory $\mathbb{W}_{\text {was }}$ published in Ref. B. The effect of finite divergence on thick grating performance has been previously described in Refs. 7 and 5 . This paper extends the results of the previous works to a modeling of the diffraction on reflecting volume gratings (Bragg mirrors) describing simultaneously divergent and polychromatic beams where Kogelnik's theory is reduced to practical formulae which are necessary for design of diffractive optical elements based on VBGs.

A uniform phase grating is a structure produced by periodical distribution of the refractive index modulation inside the volume of a photosensitive medium, as shown in Fig. 1. Each structure recorded inside a slab with plane-parallel surfaces could be considered as either transmitting or reflecting Bragg grating depending on the orientation of a readout beam in regard to the front surface. Dotted and dashed arrows in Fig. 11 correspond to a beam tracing for transmitting and reflecting grating geometry; solid arrows are the wave vectors of beams and the grating vectors. As was noted in Ref. B, uniform sinusoidal VBGs could be entirely described by the following set of parameters: the grating thickness $t$, the average refractive index of a medium $n_{\mathrm{av}}$, the amplitude of refractive index modulation $\delta n$, the grating period $\Lambda$ (or spatial frequency $f=1 / \Lambda)$, and the grating inclination angle $\varphi$ between the normal to the grating front surface $\mathbf{N}_{\mathbf{f}}$ and the grating vector $\mathbf{K}_{\mathbf{G}}$. 


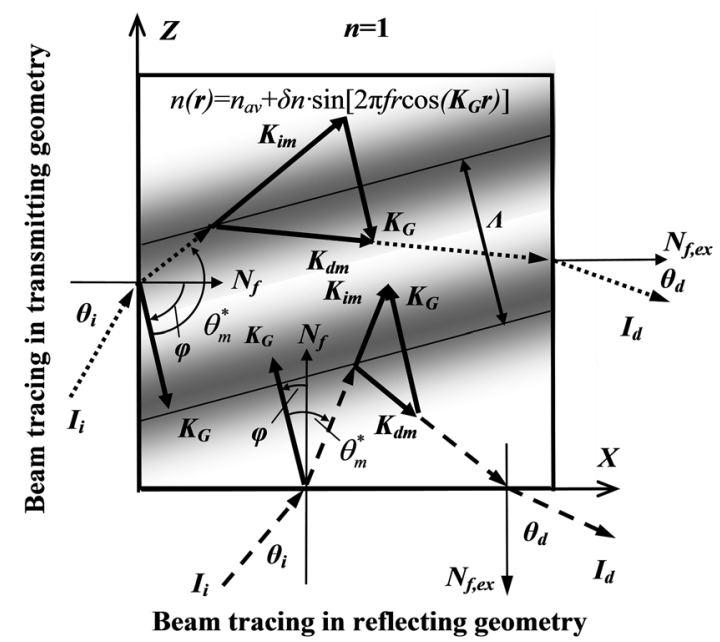

Fig. 1 Propagation of optical rays through a volume Bragg grating in transmitting (dotted lines) and reflecting (dashed lines) geometry. $\mathbf{N}_{\mathbf{f}}$ and $\mathbf{N}_{\mathrm{f}, \mathrm{ex}}=$ normal to the front surface for incident $\left(I_{i}\right)$ and diffracted $\left(I_{d}\right)$ beams; $\mathbf{K}_{\mathrm{im}}, \mathbf{K}_{\mathrm{dm}}=$ wave vectors of incident and diffracted beams inside the grating medium; $\mathbf{K}_{\mathbf{G}}=$ Grating vector; $\varphi=$ grating inclination; $\theta_{i}, \theta_{d}=$ incident and diffraction angles; $\theta_{m}^{*}=$ incident Bragg angle.

The grating vector $\mathbf{K}_{\mathbf{G}}$ is normal to the planes of a constant refractive index, has a module $\left|\mathbf{K}_{\mathbf{G}}\right|=2 \pi f$, and is directed inside a medium. It is important to emphasize that a sinusoidal volume grating itself has a double degeneration of grating vector directions due to its symmetry. Therefore, the definition of a grating vector in a diffractive optical element is based on its orientation with respect to the front surface of the element, though the process of diffraction does not depend on what surface was crossed by an incident beam. This feature of sinusoidal gratings results in different orientation of grating vector depending on what surface of a photosensitive plate was chosen as a front one. For example, according to the definition given above (introduced by Kogelnik ${ }^{\text {}}$, the volume grating depicted in Fig. 1 is a transmitting grating for short-wavelength radiation (large wave vector) which crosses the left vertical surface (front surface for this beam). The vector of this grating for this beam is directed down to the bottom of this figure. The same grating is a reflecting one for long-wavelength radiation (small wave vector) which crosses the bottom surface (front surface for this beam). The vector of this grating for this beam is directed to the top of the figure. An inclination angle of grating $\varphi$ is the angle between the normal to the front surface $\mathbf{N}_{\mathbf{f}}$ and the grating vector $\mathbf{K}_{\mathbf{G}}$. It is positive in a counter-clockwise direction and can vary from $-\pi / 2$ to $+\pi / 2$. Therefore, a transmitting grating excited through the left vertical side has a negative inclination. The same grating excited through the bottom horizontal side is a reflective one and has a positive inclination.

Following the approach proposed in Refs. 1 and 3 , we use the incident Bragg angle in a medium $\theta_{m}^{*}$, which is the angle between a grating vector $\mathbf{K}_{\mathbf{G}}$ and a wave vector $\mathbf{K}_{\mathbf{i m}}$ of an incident beam inside the medium. Let us note again that incident Bragg angle $\theta_{m}^{*}$ differs from a conventional Bragg angle in the media $\theta_{m}$, and the relationship between these parameters is $\sin \theta_{m}=\left|\cos \theta_{m}^{*}\right|$. While there are two orders of Bragg diffraction in an infinite sinusoidal grating, there are four possible orders of Bragg diffraction in sinusoidal grating recorded in a plane parallel plate. ${ }^{\text {G }}$ The positive orders of Bragg diffraction are for incident Bragg angle $0<\theta_{m}^{*}<+\pi$, while the negative orders are for $-\pi<\theta_{m}^{*}<0$. The forward orders of Bragg diffraction are for a module of an incident Bragg angle $\left|\theta_{m}^{*}\right|<\pi / 2$, while the backward orders are for $\left|\theta_{m}^{*}\right|>\pi / 2$. For transmitting geometry (dotted arrows) shown in Fig. 1, an incident Bragg angle is positive and $\left|\theta_{m}^{*}\right|>\pi / 2$. This means that this is a positive backward Bragg order. For the same grating that is used in a reflecting geometry (dashed arrows) for longer wavelengths, an incident Bragg angle for a beam coming

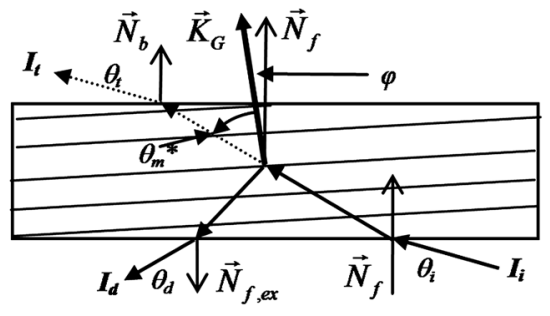

$(+)$ inclination

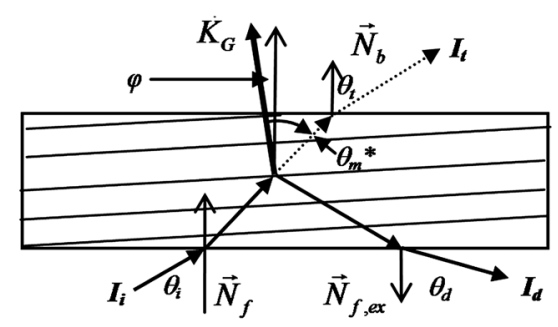

$(+)$ inclination

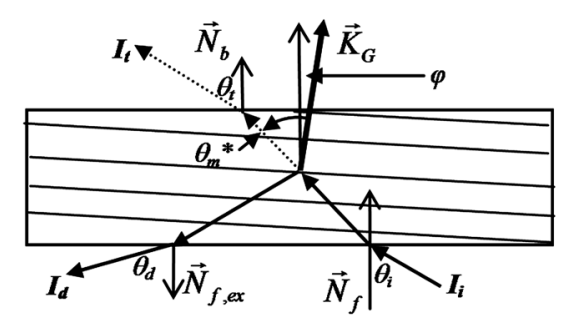

$(-)$ inclination

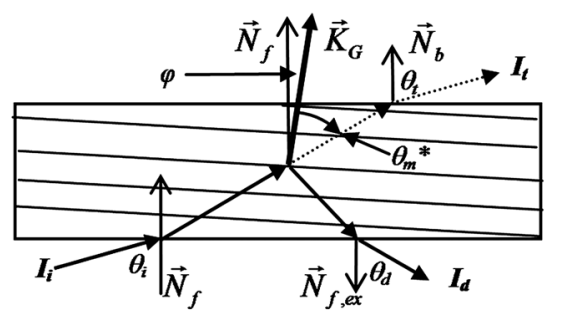

$(-)$ inclination

Fig. 2 Possible orders of Bragg diffraction for reflecting gratings with different angles of inclination. $I_{i}$, $I_{t}$, and $I_{d}=$ incident, transmitted, and diffracted beams; $\mathbf{K}_{\mathbf{G}}=$ grating vector; $\varphi=$ grating inclination; $\theta_{i}, \theta_{t}$, and $\theta_{d}=$ angles of incidence, transmission, and diffraction; $\theta_{m}^{*}=$ incident Bragg angle. 
from approximately the same direction is negative and $\left|\theta_{m}^{*}\right|<\pi / 2$; this is a negative forward Bragg order. It is important to note that for forward orders of diffraction, the wave vector of a diffracted beam $\mathbf{K}_{\mathbf{d m}}$ is the difference between the wave vector of an incident beam $\mathbf{K}_{\mathbf{i m}}$ and the grating vector $\mathbf{K}_{\mathbf{G}}$, while for backward orders the wave vector of a diffracted beam $\mathbf{K}_{\mathbf{d m}}$ is the sum of the wave vector of an incident beam $\mathbf{K}_{\mathbf{i m}}$ and the grating vector $\mathbf{K}_{\mathbf{d}}$. .

Figure 2 shows the Bragg diffraction for reflecting VBGs (Bragg mirrors) with different inclination angles $\varphi$. One can see that scanning of the incident angle results in switching between plus and minus orders only. This is the opposite of transmitting gratings, where scanning of an incident angle results in switching between forward and backward

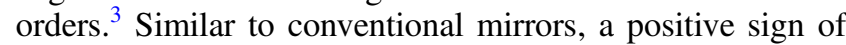
the incident angle corresponds to a negative sign of the exit diffraction angles for reflecting VBGs. Also, a change of the sign of the Bragg mirror inclination does not change the sign of a Bragg order.

Regardless of the VBG geometry, the basic relationship between Bragg wavelength $\lambda_{0}$ and incident Bragg angle $\theta_{m}^{*}$ is determined by the Bragg condition as

$\frac{\lambda_{0}}{n_{\mathrm{av}}\left|\cos \theta_{m}^{*}\right|}=2 \Lambda$.

Contrary to transmitting gratings, where $\left|\theta_{m}^{*}\right|$ is close to $\pi / 2$ and the wavelength of reading radiation is significantly less in comparison to the grating period, for reflecting gratings $\theta_{m}^{*}$ is close to zero and the wavelength of reading radiation is comparable to the grating period. For each grating period, there is the maximum wavelength $\lambda_{0}^{\max }$, which corresponds to normal beam incidence on a reflecting Bragg grating, that is, at $\theta_{m}^{*}=0$,

$\lambda_{0}^{\max }=2 n_{\mathrm{av}} \Lambda$.

Radiation with wavelength exceeding $\lambda_{0}^{\max }$ cannot be diffracted by this particular grating at any conditions of excitation. Radiation with shorter wavelengths can be reflected by a Bragg mirror at larger angles of incidence.

\section{Diffraction of Plane Monochromatic Waves on a Transmitting Bragg Grating}

Diffraction efficiency (DE) of a reflecting Bragg grating that has no material losses is described by the following formula if $\xi$ and $S$ are considered to be real positive numbers and $\xi>$ $S$ (Ref. (1). This equation also appears in the theory for fiber Bragg gratings.

$\eta=\frac{\sin ^{2} \sqrt{\xi^{2}-S^{2}}}{\xi^{2} / S^{2}-\cos ^{2} \sqrt{\xi^{2}-S^{2}}}$.

Here $S$ and $\xi$ are the same phase incursion, also called grating strength, at Bragg condition and dephasing parameter at certain detuning from Bragg condition as they were described in Ref. 3 for transmitting gratings. However, there is a distinctive feature between transmitting and reflecting gratings: the inclination factor, $F_{\varphi}=\left[-\cos \left(\varphi-\theta_{m}^{*}\right) \cos \left(\varphi+\theta_{m}^{*}\right)\right]^{1 / 2}$, is a real number for transmitting gratings and an imaginary number for reflecting ones because the incident Bragg angle $\theta_{m}^{*}$ is close to $\pi / 2$ and to zero, respectively. Therefore, in order to maintain the phase incursion $S$ as a real-valued parameter, it should be redefined for reflecting VBGs as:

$S=\frac{i \pi t \delta n}{\lambda_{0} F_{\varphi}}$

For unslanted reflecting grating $\varphi=0$, and the phase incursion $S$ becomes:

$S_{0}=\frac{\pi t \delta n}{\lambda_{0}\left|\cos \theta_{m}^{*}\right|}=\frac{2 \pi n_{\mathrm{av}} t \delta n}{\lambda_{0}^{2} f}$.

Similarly, when small angular deviations $\Delta \theta_{m}$ from an incident Bragg angle $\theta_{m}^{*}$ and/or small deviations $\Delta \lambda$ from central wavelength $\lambda_{0}$ occurred, dephasing parameter $\xi$ for reflecting gratings should be redefined for keeping it positive by changing of the sign in Eq. (6). The resulting dephasing parameter becomes:

$\xi=\frac{\pi f t \cos \left(\theta_{m}^{*}\right)}{\cos \left(\varphi-\theta_{m}^{*}\right)-\frac{f \lambda_{0}}{n_{\mathrm{av}}} \cos (\varphi)}\left[\Delta \theta_{m} \frac{\sin \left(\theta_{m}^{*}\right)}{\cos \left(\theta_{m}^{*}\right)}+\frac{\Delta \theta_{m}^{2}}{2}+\frac{\Delta \lambda}{\lambda_{0}}\right]$.

Kogelnik's dephasing parameter, $-\Delta \theta_{m} \sin \left(\theta_{m}^{*}\right)+\frac{f}{2 n_{\mathrm{av}}} \Delta \lambda$, is the result of a first-order Taylor series approximation of $\cos \left(\theta_{m}^{*}+\Delta \theta_{m}\right)+\frac{f}{2 n_{\text {av }}}\left(\lambda_{0}+\Delta \lambda\right)$. However, this approximation will not be valid if the Bragg angle is set to near normal incidence, as $\sin \left(\theta_{m}^{*}\right) \approx 0$ in this case. Therefore, one has to use the second term in the approximation.

Let us assume that for unslanted $(\varphi=0)$ reflecting VBGs the incident beam corresponds to the angular Bragg condition (i.e., $\Delta \theta_{m}=0$ ), but there is some spectral deviation $\Delta \lambda$ from exact Bragg wavelength $\lambda_{0}$. In this case, one can write a formula for dephasing parameter $\xi$ from Eq. (6) by considering the Bragg condition [Eq. (1)]:

$\xi_{0}=\frac{2 \pi n_{\mathrm{av}} t \Delta \lambda}{\lambda_{0}^{2}}\left|\cos \theta_{m}^{*}\right|=\frac{\pi f t \Delta \lambda}{\lambda_{0}}$.

Spectral selectivity of such unslanted grating could be derived by substitution of Eqs. (5) and (ד) to Eq. (3)

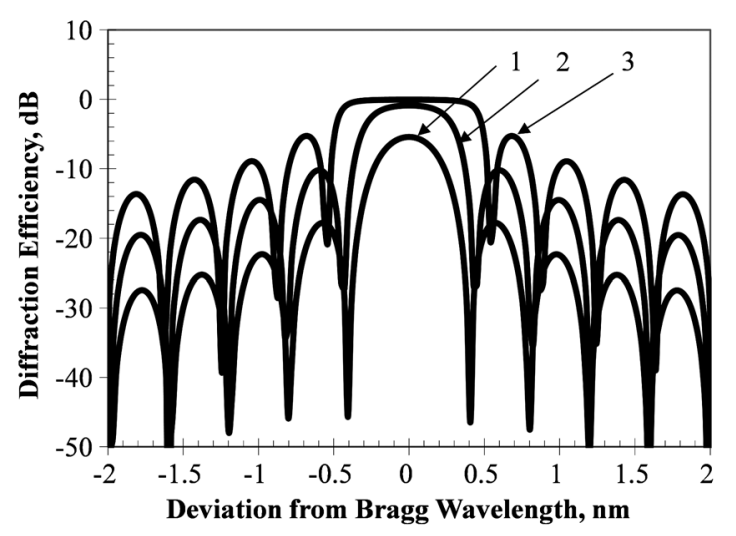

Fig. 3 Dependence of diffraction efficiency of a reflecting VBG on deviation from resonant wavelength $\lambda_{0}=1085 \mathrm{~nm}$. Normal incidence, thickness $1 \mathrm{~mm}$, average refractive index $n_{\mathrm{av}}=1.4867$. Refractive index modulation, ppm: $1=200,2=500,3=1000$. 
when the mismatching from the exact Bragg wavelength $\lambda_{0}$ of $\Delta \lambda$ occurs. The selectivity could be described in the terms of Bragg grating parameters:

$$
\eta=\frac{\sin ^{2} \sqrt{\left(\frac{2 \pi n_{\mathrm{av}} t \delta n}{\lambda_{0}^{2} f}\right)^{2}-\left(\frac{\pi f t \Delta \lambda}{\lambda_{0}}\right)^{2}}}{\left(\frac{\lambda_{0} f^{2} \Delta \lambda}{2 n_{\mathrm{av}} \delta n}\right)^{2}-\cos ^{2} \sqrt{\left(\frac{2 \pi n_{\mathrm{av}} t \delta n}{\lambda_{0}^{2} f}\right)^{2}-\left(\frac{\pi f t \Delta \lambda}{\lambda_{0}}\right)^{2}}} .
$$

Figure 3 shows the dependence of diffraction efficiency (reflection coefficient) on spectral detuning from Bragg wavelength for 1-mm-thick reflecting Bragg grating for three different values of refractive index modulation $\delta n$. If $\delta n$ is decreasing, the spectral selectivity is narrowing and the maximal DE decreases. A reflecting VBG at Bragg condition $\left(\Delta \theta_{m}=\Delta \lambda=0\right)$ has a dephasing parameter $\xi=0$, and the maximal DE can be simplified from Eq. (3):

$\eta_{0}=\tanh ^{2} S_{0}=\tanh ^{2} \frac{\pi t \delta n}{\lambda_{0}\left|\cos \theta_{m}^{*}\right|}$.

Following the hyperbolic tangent function, diffraction efficiency $\eta_{0}$ asymptotically approaches the $100 \%$ value by increasing of grating thickness and/or refractive index modulation.

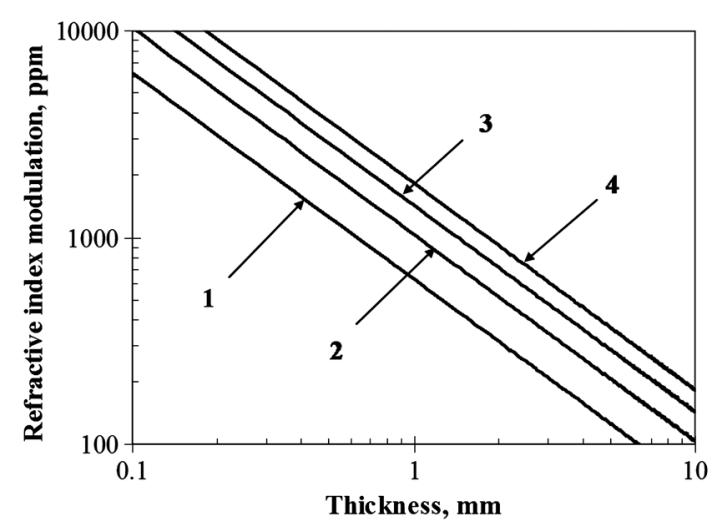

(a)

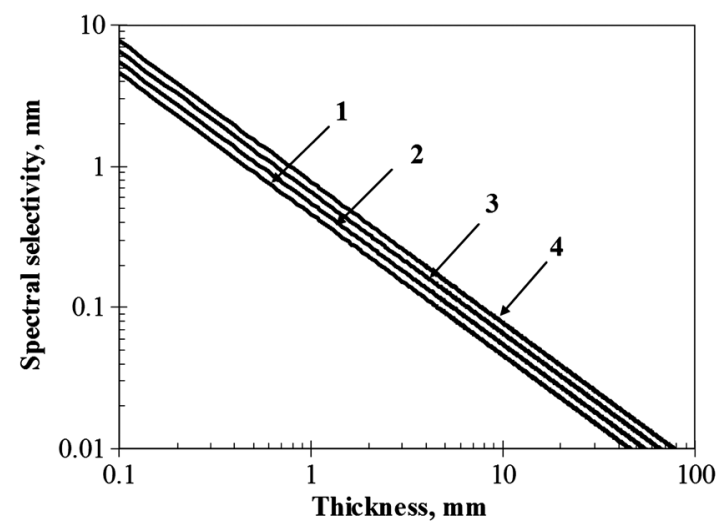

(b)

Fig. 4 Interrelationship between grating parameters and spectral selectivity for predetermined diffraction efficiency of VBG: $1=90 \%$, $2=99 \%, 3=99.9 \%, 4=99.99 \%$. Normal incidence, $\lambda_{0}=1085 \mathrm{~nm}$, $n_{\mathrm{av}}=1.4867$. (a) Refractive index modulation versus thickness. (b) Spectral selectivity at HWFZ versus thickness.
Let us note that if the desired diffraction efficiency $\eta_{0}$ could be predetermined at a certain level, it would serve as one of the basic parameters for a design of a reflecting VBG. To achieve this specified diffraction efficiency, interrelationships between thickness, refractive index modulation, and incident Bragg angle $\theta_{m}^{*}$ could be derived from Eq. (9):

$\delta n=\frac{\lambda_{0}\left|\cos \theta_{m}^{*}\right| \operatorname{atanh} \sqrt{\eta_{0}}}{\pi t_{0}}$.

Figure $4(\mathrm{a})$ illustrates this interrelation [Eq. (10)] for four DE values: $90 \%$, which corresponds to $10 \mathrm{~dB}$ of attenuation of a transmitted beam; $99 \%(20 \mathrm{~dB}) ; 99.9 \%(30 \mathrm{~dB})$; and $99.99 \%$ (40 dB), at $\lambda_{0}=1085 \mathrm{~nm}$ for $\theta_{m}^{*}=0$ (normal incidence). It can be noted that refractive index modulation $\delta n$ becomes less than $1000 \mathrm{ppm}$ for securing the diffraction efficiency level of $\eta_{0}=99 \%$ (20 dB attenuation) only when a VBG thickness is more than $1 \mathrm{~mm}$. Therefore, reflecting VBGs should be thick enough to achieve efficient reflection at relatively low values of refractive index modulation.

One can conclude that unslanted reflecting VBGs can be fully described by a combination of any two out of three grating parameters: thickness, refractive index modulation, and diffraction efficiency that should be predetermined for a particular incident Bragg angle $\theta_{m}^{*}$ at a wavelength $\lambda_{0}$.

To determine spectral selectivity, Eq. (3) should be equaled to zero. Because $\xi \neq S$ (otherwise we have a function singularity at this point), diffraction efficiency reaches its zero value at multiple points:

$$
\left(\xi^{2}-S^{2}\right)^{1 / 2}=j \pi, \quad \text { where } j=1,2, \ldots, n, \ldots
$$

Generally, spectral selectivity at a half width at first zero (HWFZ) level, $\delta \lambda^{\mathrm{HWFZ}}$, is defined by substitution of Eqs. (\#) and (6) into Eq. (11) at $j=1$. However, the general result can be simplified for unslanted reflecting VBGs with maximal DE $\eta_{0}$ by substitution of $S_{0}$ and $\xi_{0}$ from Eqs. (7) and (9) to Eq. (11):

$$
\begin{aligned}
\delta \lambda^{\mathrm{HWFZ}} & =\frac{\lambda_{0}^{2}\left[\left(\operatorname{atanh} \sqrt{\eta_{0}}\right)^{2}+\pi^{2}\right]^{1 / 2}}{2 \pi n_{\mathrm{av}} t\left|\cos \theta_{m}^{*}\right|} \\
& =\frac{\lambda_{0}\left[\left(\operatorname{atanh} \sqrt{\eta_{0}}\right)^{2}+\pi^{2}\right]^{1 / 2}}{\pi f t} .
\end{aligned}
$$

Assessment of typical values of spectral selectivity $\delta \lambda^{\mathrm{HWFZ}}$ for $\eta_{0}=99.0 \%, \lambda_{0}=1085 \mathrm{~nm}, n_{\mathrm{av}}=1.485$, and $\theta_{m}^{*}=0$ gives the following formula:

$\delta \lambda^{\mathrm{HWFZ}}[\mathrm{nm}] \cong \frac{0.55}{t[\mathrm{~mm}]}$.

It is clear that this simple expression can be easily derived for any particular wavelength. It is necessary to point out that refractive index modulation $\delta n$ has to follow any changing of thickness for securing the diffraction efficiency value of $\eta_{0}$. Figure 4(b) illustrates the interrelation between spectral selectivity $\delta \lambda^{\mathrm{HWFZ}}$ and grating thickness $t$ that is necessary to achieve for different diffraction efficiency values: the 
thicker the grating, the narrower the spectral selectivity. One can see that a spectral selectivity $\delta \lambda^{\mathrm{HWFZ}} \sim 0.1 \mathrm{~nm}$ could be achieved for a 5.5-mm-thick VBG, and $\delta \lambda^{\mathrm{HWFZ}} \sim$ $0.5 \mathrm{~nm}$ corresponds to a $1.1-\mathrm{mm}$-thick grating at $\eta_{0}=99 \%$. The coefficient in Eq. (13) slightly increases for higher DE values.

Let us derive the basic interrelation between angular and spectral parameters for a reflecting VBG. Similarly to the procedure described in Refs. 11 and 2 for transmitting Bragg gratings, we suppose that small angular deviation $\Delta \theta$ from $\theta_{m}^{*}$ inside the grating medium leads to corresponding change $\Delta \lambda$ in resonant wavelength $\lambda_{0}$. In this case, Bragg condition, Eq. (II), can be expressed in its differential form:

$$
\frac{\lambda_{0}-\Delta \lambda}{\lambda_{0}}=\left|\frac{\cos \left(\theta_{m}^{*}-\Delta \theta\right)}{\cos \theta_{m}^{*}}\right| .
$$

Because deviation $\Delta \theta$ from $\theta_{m}^{*}$ is small, $\cos \Delta \theta=$ $1-2 \sin ^{2} \frac{\Delta \theta}{2} \approx 1-\frac{\Delta \theta^{2}}{2}$, and Eq. (14) becomes

$$
\Delta \theta^{2}-2 \tan \theta_{m}^{*} \Delta \theta-\frac{2 \Delta \lambda}{\lambda_{0}}=0 .
$$

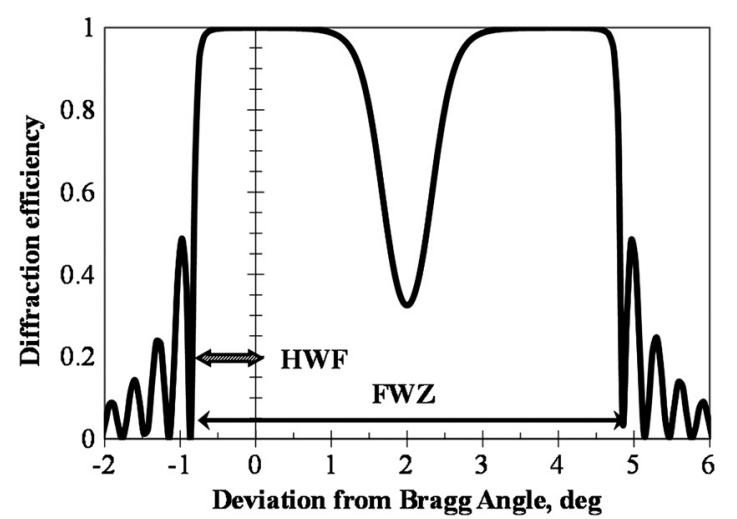

(a)

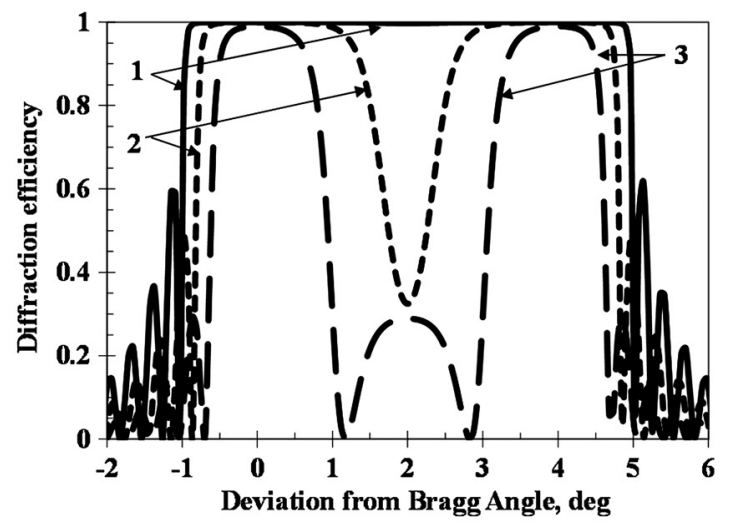

(b)

Fig. 5 Angular selectivity of $1-\mathrm{mm}$-thick reflecting VBG at $\theta_{m}^{*}=2^{\circ}$. $\lambda_{0}=1085 \mathrm{~nm}, n_{\mathrm{av}}=1.4867$. (a) Definition of angular selectivity at HWFZ (solid arrow) and FWZ (dashed arrow) levels. (b) Dependence of angular selectivity shape on refractive index modulation: $1=$ 2000 ppm (solid line), $2=1500$ ppm (dotted line), $3=1000$ ppm (dashed line).
Equation (15) has two solutions:

$\Delta \theta_{1,2}= \pm\left(\tan ^{2} \theta_{m}^{*}+\frac{2 \Delta \lambda}{\lambda_{0}}\right)^{1 / 2}+\tan \theta_{m}^{*}$

If one would like to consider angular selectivity between the first zeros of the DE, it is necessary to substitute $\delta \lambda^{\mathrm{HWFZ}}$ from Eq. (12) to Eq. (16). However, Eq. (15) has two different solutions and we have to introduce two different definitions for angular selectivity. Figure 5(a) shows the simulation results for angular selectivity of a $1-\mathrm{mm}$-thick VBG with 1500 ppm refractive index modulation and an incident Bragg angle of $2 \mathrm{deg}$ at $1085 \mathrm{~nm}$. The first definition is for full (wider) angular selectivity at full width at zero (FWZ) level, $\delta \theta_{m}^{\mathrm{FWZ}}$, i.e., for the distance between two first zeros in angular selectivity as they are determined by both solutions of Eq. (15):

$\delta \theta_{m}^{\mathrm{FWZ}}=\Delta \theta_{2}-\Delta \theta_{1}=2\left(\tan ^{2} \theta_{m}^{*}+\frac{2 \delta \lambda^{\mathrm{HWFZ}}}{\lambda_{0}}\right)^{1 / 2}$

Full angular selectivity $\delta \theta_{m}^{\mathrm{FWZ}}$ of reflecting VBGs with predetermined DE $\eta_{0}$ and incident Bragg angle $\theta_{m}^{*}$ could be written in the following form by substitution of the spectral selectivity value from Eq. (12):

$\delta \theta_{m}^{\mathrm{FWZ}}=2\left[\tan ^{2} \theta_{m}^{*}+\frac{2 \sqrt{\left(\operatorname{atanh} \sqrt{\eta_{0}}\right)^{2}+\pi^{2}}}{\pi f t}\right]^{1 / 2}$.

Let us note that full angular selectivity $\delta \theta_{m}^{\mathrm{FWZ}}$ should be the primary consideration when incident Bragg angles $\theta_{m}^{*}$ are small enough. In this case, an overlapping of the two different selectivity curves has occurred due to their proximity, and a divergent incident beam can interact with both of them. Angular selectivity $\delta \theta_{m}^{\mathrm{FWZ}}$ can be affected by changing of refractive index modulation, especially for overdeveloped VBGs. Figure 5(b) shows this phenomenon for 1-mmthick grating at $\theta_{m}^{*}=2^{\circ}$ incident Bragg angle. One can see that slight overall widening of $\delta \theta_{m}^{\mathrm{FWZ}}$ is accompanied with dramatically changing of angular selectivity shape. The shape is changing from total overlapping of diffraction peaks at more than $99.99 \%$ diffraction efficiency (which corresponds to refractive index modulation $\delta n=2000 \mathrm{ppm}$ ) to partial peak separating at $\eta_{0} \approx 99 \%(\delta n=1500 \mathrm{ppm})$ and their full dividing at $\eta_{0} \approx 99 \%(\delta n=1000 \mathrm{ppm})$. This peculiarity of reflecting VBGs should be considered for $\delta \theta_{m}^{\mathrm{FWZ}}$ assessment when gratings' DE is near the $100 \%$ diffraction efficiency limit.

The second definition of VBG angular selectivity is for the distance between the Bragg angle and the first zero closest to it [Fig. [(a)]. Let us call this reduced angular selec-

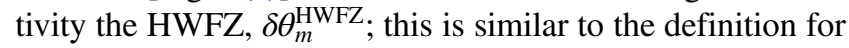
transmitting VBGs introduced in Ref. 3. The HWFZ angular selectivity considers the negative solution of Eq. (15) and assumes that it has more universal nature and can be used for reflecting Bragg gratings with any diffraction efficiency and/or incident Bragg angle $\theta_{m}^{*}$, especially when the selectivity curves corresponding two different diffraction orders do not overlap. The analytical formula for the HWFZ selectivity is 
$\delta \theta_{m}^{\mathrm{HWFZ}}=\left|\Delta \theta_{1}\right|=\left(\tan ^{2} \theta_{m}^{*}+\frac{2 \delta \lambda^{\mathrm{HWFZ}}}{\lambda_{0}}\right)^{1 / 2}-\tan \theta_{m}^{*}$.

For unslanted gratings $\theta_{m}^{*}=0$, and the interrelation between both definitions of angular selectivity can be significantly simplified:

$\delta \theta_{m}^{\mathrm{FWZ}}=2 \delta \theta_{m}^{\mathrm{HWFZ}}=\left(\frac{8 \delta \lambda^{\mathrm{HWFZ}}}{\lambda_{0}}\right)^{1 / 2}$.

Because there are two different definitions of Bragg grating angular selectivity, we should determine the applicability range for both definitions. Let us estimate at what incident Bragg angle $\theta_{m}^{*}$ one should consider the FWZ angular selectivity rather than the doubled HWFZ value. This problem is very close to the problem of separation of two functions with two maxima located near each other. Historically, three main criteria for such separation were developed by Lord Rayleigh (1879), C. M. Sparrow (1916), and W. V. Houston (1926). The Rayleigh criterion states that the separation arises when the peak of one function falls on the first zero point of the other function (so-called null first derivative criterion); the Sparrow criterion defines separation as when the saddle point of the summed function first develops (so-called null second derivative criterion); the Houston criterion states that the intersection of two identical functions at their half maximum level is a suitable definition for their effective separation.

Let us apply the classical Rayleigh criterion, because the Sparrow criterion is too strict and Houston's does not work well for functions which have varying core to wing ratios. 4

Let us call the incident Bragg angle that defines the fulfillment of Rayleigh's criterion as the threshold incident Bragg angle $\theta_{0}$. To determine it, the following procedure should be applied. Actually, $\theta_{0 m}$ is the angle inside the VBG medium at which full FWZ angular selectivity is three times wider than HWFZ selectivity: $\delta \theta_{m}^{\mathrm{FWZ}}=$ $3 \delta \theta_{m}^{\mathrm{HWFZ}}$. Combining of Eqs. (18) and (19) together with Eq. (12) gives a result

$\tan ^{2} \theta_{0}=\frac{\delta \lambda^{\mathrm{HWFZ}}}{4 \lambda_{0}}=\frac{\lambda_{0}\left[\left(a \tanh \sqrt{\eta_{0}}\right)^{2}+\pi^{2}\right]^{1 / 2}}{8 \pi n_{\mathrm{av}} t\left|\cos \theta_{0}\right|}$.

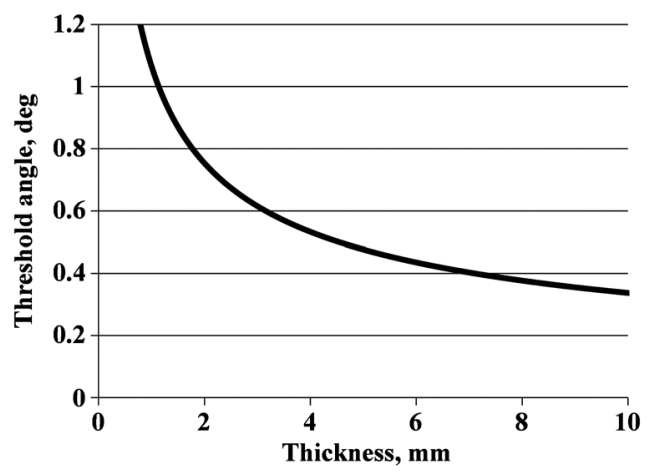

Fig. 6 Dependence of threshold incident angle in air on thickness of reflecting VBG with $99.9 \%$ diffraction efficiency at resonant wavelength of $\lambda_{0}=1085 \mathrm{~nm}$.

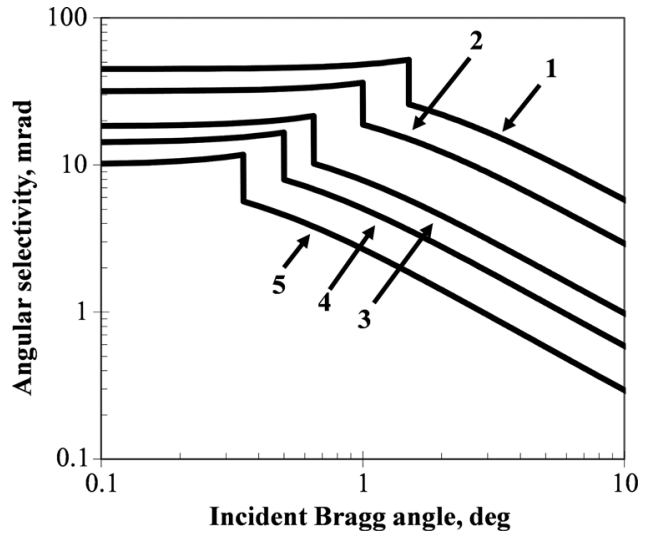

Fig. 7 Angular selectivity of reflecting VBG with 99\% diffraction efficiency at wavelength $\lambda_{0}=1085 \mathrm{~nm}$ versus incident Bragg angle in the medium at different grating thickness, $\mathrm{mm}: 1=0.5 ; 2=1$; $3=3 ; 4=5 ; 5=10$.

One can easily calculate the $\theta_{0}$ angle as an angle outside the VBG (i.e., in air). As an example, it is about $1 \mathrm{deg}$ for $t=1 \mathrm{~mm}$ and $\eta_{0}=99.9 \%$ for $\lambda_{0}=1085 \mathrm{~nm}$. The dependence of the threshold angle in air on VBG thickness is shown in Fig. 6. The thinner the grating, the bigger the threshold angle is. However, differences in diffraction efficiency of relatively strong reflecting VBGs have almost no influence on the shape of this curve and the values of threshold angles.

Hence, at $\theta_{m}^{*}<\theta_{0}$ one should define the angular selectivity of reflecting VBG as its FWZ value, $\delta \theta_{m}^{\mathrm{FWZ}}$; otherwise, a doubled value of the HWFZ selectivity, $\delta \theta_{m}^{\mathrm{HWFZ}}$, should be taken into consideration at $\theta_{m}^{*}>\theta_{0}$.

Figure 7 shows the dependence of angular selectivity on VBG thickness at different incident angles for a $99 \%$ efficient grating. As one can see, the thicker the grating (still in the assumption that all gratings are $99 \%$ efficient by respective choosing of their refractive index modulations), the wider the angular selectivity is. At normal incidence $\left(\theta_{m}^{*}=0\right)$, angular selectivity is $20 \mathrm{mrad}$ for $10-\mathrm{mm}$-thick, $28 \mathrm{mrad}$ for 5 -mm-thick, and $65 \mathrm{mrad}$ for 1 -mm-thick gratings with a $1085 \mathrm{~nm}$ resonant wavelength. When incident angles are small $\left(\theta_{m}^{*}<\theta_{0}\right)$, angular selectivity is slightly wider due to the appearance of two separated diffraction maxima and their partial overlapping. The relative amount of this widening is higher for thicker gratings. In these conditions, reflecting VBGs work well for spectral narrowing and mode-locking of diode lasers, $\mathbb{0}$ as a coupling element in laser resonators. 10,

Due to separation of two maxima of angular selectivity according to Rayleigh criterion at $\theta_{m}^{*}=\theta_{0}$, the FWZ definition of angular selectivity of reflecting VBG should be replaced by its HWFZ definition. Figure 7 shows the corresponding drop in VBG selectivity at this point that was determined from Eq. (21). Then, the angular selectivity dramatically narrows with increasing of incident angle at $\theta_{m}^{*}>\theta_{0}$; however, even at high angles of incidence angular selectivity of reflecting VBGs is still much wider in comparison with typical transmitting VBGs. Possible applications of VBGs in non-retroreflecting condition are coherent and spectral beam combining, deflecting, splitting, and attenuating of laser beams, etc 13

Hence, despite its spectral selectivity being unambiguously specified, the angular selectivity of a reflecting 
Bragg grating has two different definitions due to partial (or full) overlapping of positive and negative orders of Bragg diffraction. This phenomenon is inherent to reflecting Bragg gratings and it could be used for design of high-selective spectral filters with relatively wide angular selectivity.

\section{Diffraction of Gaussian Beams by Reflecting VBGs}

\subsection{Polychromatic Planar Waves}

Similarly to diffraction of Gaussian beams on transmitting Bragg gratings described in Ref. 1, we will consider the Bragg diffraction by comparing of grating spectral selectivity with the beam spectrum:

$G_{1}(\Delta \lambda, w)=e^{-2\left(\frac{\Delta \lambda-\lambda_{0}}{w}\right)^{2}}$,

where parameter $w$ is the half width at $e^{-2}$ of the maximum $\left(\mathrm{HWe}^{-2} \mathrm{M}\right)$ spectral width and $\lambda_{0}$ is a central wavelength of a

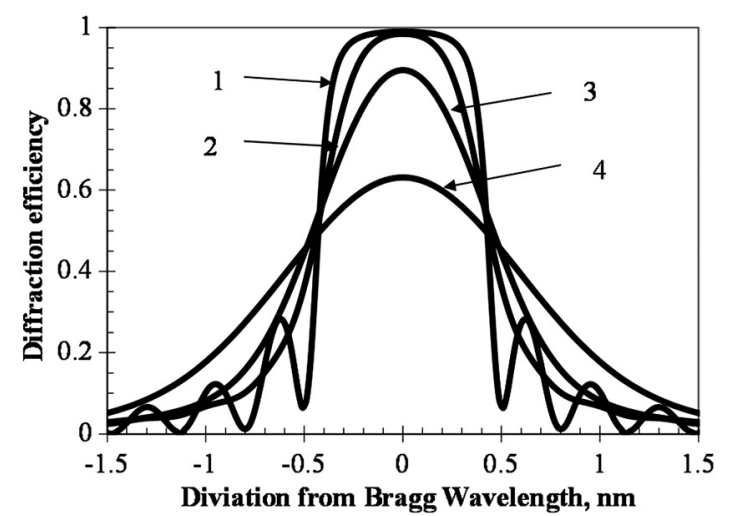

(a)

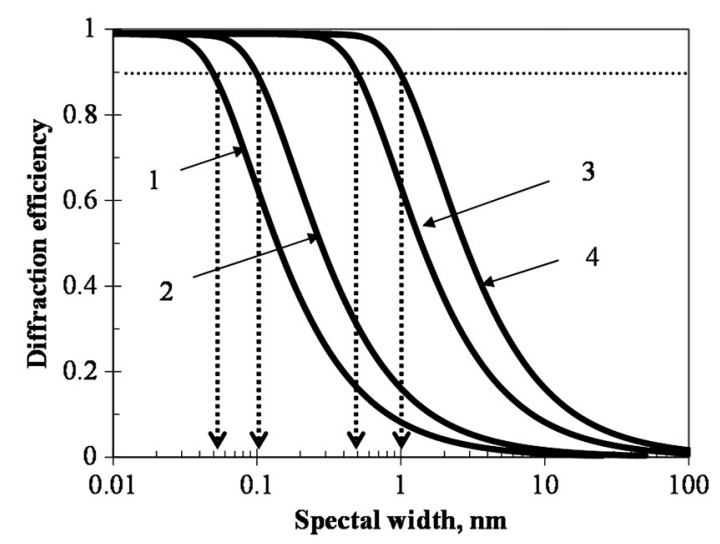

(b)

Fig. 8 Spectral selectivity of $99 \%$ efficient reflecting VBG for polychromatic plane waves at normal incidence. The calculated VBG is $1.1 \mathrm{~mm}$ thick with approximately 1000 ppm refractive index modulation. (a) Dependence of diffraction efficiency on deviation from Bragg wavelength. Spectral width of a beam $\left(\mathrm{HWe}^{-2} \mathrm{M}\right), \mathrm{nm}: 1=0.05$, $2=0.25,3=0.5,4=1.0$; grating spectral selectivity (HWFZ) is $0.5 \mathrm{~nm}$. (b) Dependence of grating DE on spectral width of the beam. Spectral selectivity of gratings (HWFZ), $\mathrm{nm}: 1=0.05$, $2=0.1,3=0.5,4=1.0$; shown by dotted arrows. Dotted line corresponds to diffraction efficiency for a beam with spectral width which is equal to the grating selectivity. beam emission. In this case, grating diffraction efficiency spectrum could be calculated from a convolution of the grating DE for monochromatic wave, Eq. (8), with the Gaussian spectral distribution, Eq. (22). The convolution is normalized with the integral of the Gaussian bandwidth profile. This gives us the adjusted value of diffraction efficiency $\eta_{\lambda}(w)$ as a function of wavelength.

$\eta_{\lambda}(\Delta \lambda, w)=\frac{\left[\eta * G_{1}\right](\Delta \lambda)}{\int G_{1}(\Delta \lambda, w) \mathrm{d} \Delta \lambda}=\frac{\int \eta\left(\lambda_{c}\right) G_{1}\left(\Delta \lambda-\lambda_{c}\right) \mathrm{d} \lambda_{c}}{\int G_{1}(\Delta \lambda, w) \mathrm{d} \Delta \lambda}$

Results of numerical calculations of Bragg diffraction on reflecting gratings are shown in Fig. 8. Figure 8(a) shows how different polychromatic beams diffract on a 1.1-mmthick grating which has $99 \%$ diffraction efficiency at normal beam incidence. According to Eq. (12), this VBG has $0.5 \mathrm{~nm}$ of HWFZ spectral selectivity. One can see that diffraction efficiency drops down as the beam spectrum widens; this grating has diffraction efficiency of about $60 \%$ when $w=2 \delta \lambda^{\mathrm{HWFZ}}$. In comparison with the same dependence of spectral selectivity for transmitting gratings described in Ref. 11, when $60 \%$ DE occurs at $w=\delta \lambda^{\mathrm{HWFZ}}$, one can conclude that spectral width of incident beams is half as restrictive of a parameter for reflecting VBGs in comparison with transmitting ones. Also, the side lobes in the spectral selectivity curves begin to disappear when the beam spectral width is approximately half the grating spectral selectivity $\left(2 w \geq \delta \lambda^{\mathrm{HWFZ}}\right)$; total flattening of the DE curve occurs when these values become equal to each other and for further increasing of the beam spectral linewidth $\left(w \geq \delta \lambda^{\mathrm{HWFZ}}\right)$.

Figure 8(b) shows the dependence of peak diffraction efficiency $\eta \lambda(\Delta \lambda=0, w)$ on the beam spectral bandwidth $w$ for a set of VBGs with different spectral selectivity. These gratings have thickness of $10.9,5.5,1.1$, and $0.55 \mathrm{~mm}$, and all are 99\% efficient for monochromatic wave. Their HWFZ spectral selectivity is determined from Eq. (13) as 0.05, 0.1, 0.5, and $1.0 \mathrm{~nm}$, respectively. One can see that $99 \%$ efficient reflecting VBGs exhibit losses of about $10 \%$ for beams having a spectral width equal to the spectral selectivity of a corresponding grating. It is important to note that a reflecting VBG has fewer losses in comparison with a transmitting VBG having the same spectral selectivity when a beam bandwidth is equal to the spectral selectivity of the grating. ${ }^{6}$ For reflecting VBGs, decreasing DE from $99 \%$ to $98 \%$ occurs when the beam width, $w$, becomes approximately half the grating's spectral selectivity. This parameter for $1 \%$ efficiency decreasing ratio is about four times less restrictive in comparison with the same parameter for transmitting VBG as it was determined in Ref. B.

It is important to note that the general shape of the curves showed in Fig. 8does not change when the beam incidence is not normal (i.e., incident Bragg angle $\theta_{m}^{*} \neq 0$ ) when the beam wavelength obeys the relation $\lambda_{0}=\lambda_{0}^{\max }\left|\cos \theta_{m}^{*}\right|$. Thus, one can use the criterion described above for the determination of diffraction losses resulting from spectral widening of diffracted beams for all values of incident Bragg angles $\theta_{m}^{*}$ 


\subsection{Divergent Monochromatic Beams}

Similar to the description for transmitting VBGs in Ref. 3, monochromatic beams that have a certain divergence could be approximated by a Gaussian function with $\mathrm{HWe}^{-2} \mathrm{M}$ divergence $b$ :

$G_{2}\left(\Delta \theta_{m}, b\right)=e^{-2\left(\frac{\Delta \theta_{m}-\theta_{m}^{*}}{b}\right)}$.

Diffraction of divergent beams on reflecting VBG could be described by a convolution of the grating diffraction efficiency, $\eta(\Delta \theta)$, given by Eqs. (ब) and (B), and the angular distribution of the beam. In this case, wavelength is taken to be a constant, $\Delta \lambda=0$.

$$
\begin{aligned}
\eta_{\theta}(\Delta \theta, b) & =\frac{\left[\eta * G_{2}\right]\left(\Delta \theta_{m}\right)}{\int G_{2}\left(\Delta \theta_{m}, b\right) \mathrm{d} \Delta \theta_{m}} \\
& =\frac{\int \eta\left(\theta_{c}\right) G_{2}\left[\left(\Delta \theta_{m}-\theta_{c}\right), b\right] \mathrm{d} \theta_{c}}{\int G_{2}\left(\Delta \theta_{m}, b\right) \mathrm{d} \Delta \theta_{m}} .
\end{aligned}
$$

Although the general shape of the angular selectivity for reflecting VBGs at normal incidence is the same as its spectral selectivity shown in Fig. 8(a), splitting of angular selectivity curves described above results in more complex interrelations between angular selectivity of a grating, divergence of a beam, and the resulting diffraction efficiency. Let us consider a set of Bragg gratings similar to those which were described above in Sec. 3.1. Gratings with thickness 10.8, 5.4, 1.08, and $0.54 \mathrm{~mm}$ each have a corresponding HWFZ angular selectivity of $0.26,0.53,2.6,5.2 \mathrm{mrad}$ at an incident Bragg angle in the medium of $\theta_{m}^{*}=10 \mathrm{deg}$. A 1.1-mm-thick reflecting VBG has angular selectivity of $30.3,22.8,17.5,5.1$, and $2.6 \mathrm{mrad}$ for corresponding Bragg angles of $0,0.5,1,5$, and $10 \mathrm{deg}$. FWZ angular selectivity for this grating is $30.3,31.6$, and $35.1 \mathrm{mrad}$ for incident Bragg angles of $0,0.5$, and $1.0 \mathrm{deg}$, respectively. Figure 9(a) shows the results of numerical modeling of diffraction efficiency $\eta_{\theta}(\Delta \theta, b)$ versus beam divergence $b$. One can see a rather complex dependence of diffraction efficiency on divergence of an incident beam for incident Bragg angles between the normal and threshold angle $\theta_{0}$ [see Fig. 9(b)]. Despite the fact that angular selectivity decreases considerably while the Bragg angle increases [Fig. 9(b)], diffraction efficiency reaches its maximum at $\theta_{m}^{*}=\theta_{0}$ due to overlapping of the positive and negative diffraction orders. Further increasing of incident Bragg angle results in a weak decreasing of this DE value down to a level of about $90 \%$; it is in good correlation with the data shown in Fig. 8(b). Moreover, when an incident Bragg angle well exceeds the threshold angle of $\theta_{0} \approx 1^{\circ}$, the incident beam interacts with only one diffraction order of the grating and, therefore, there is no difference between angular and spectral parameters of the grating as they are shown in Figs. 8(b) and 9(c).

It should be noticed again that DE is about $90 \%$ when beam divergence $b$ equals the angular selectivity of the grating (HWZ), and these results are similar to the those for spectral characterization of reflecting VBGs as it was discussed in Sec. 3.1. Decreasing of grating DE from $99 \%$ to $98 \%$ occurs when the beam divergence $b$ is equal to half the angular selectivity of the grating (HWM) $\left(2 b=\delta \theta_{m}\right)$. In comparison with diffraction of divergent beams on transmitting gratings described in Ref. B, this $1 \%$ loss

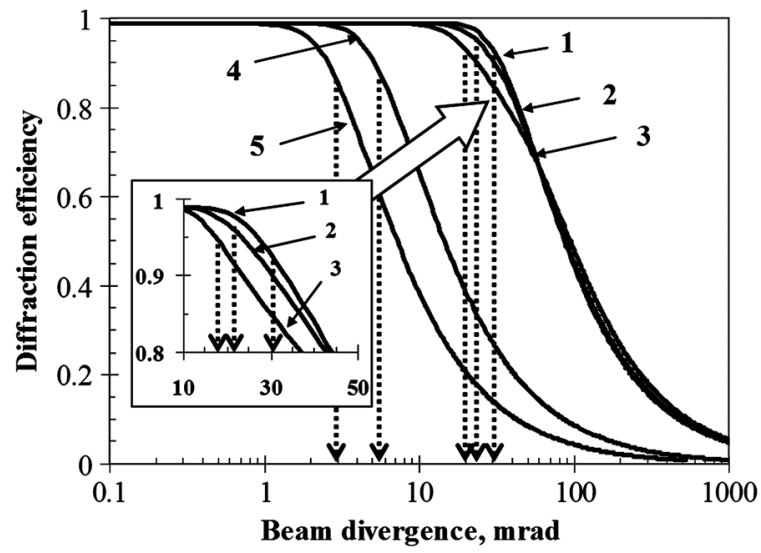

(a)

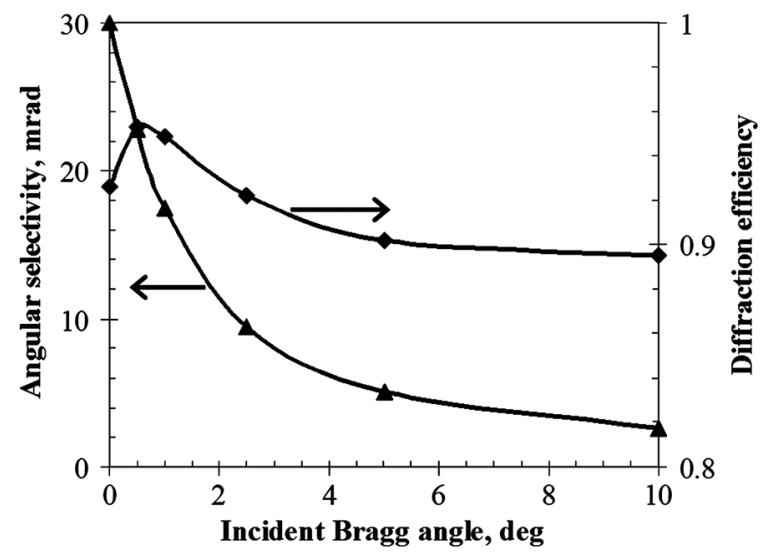

(b)

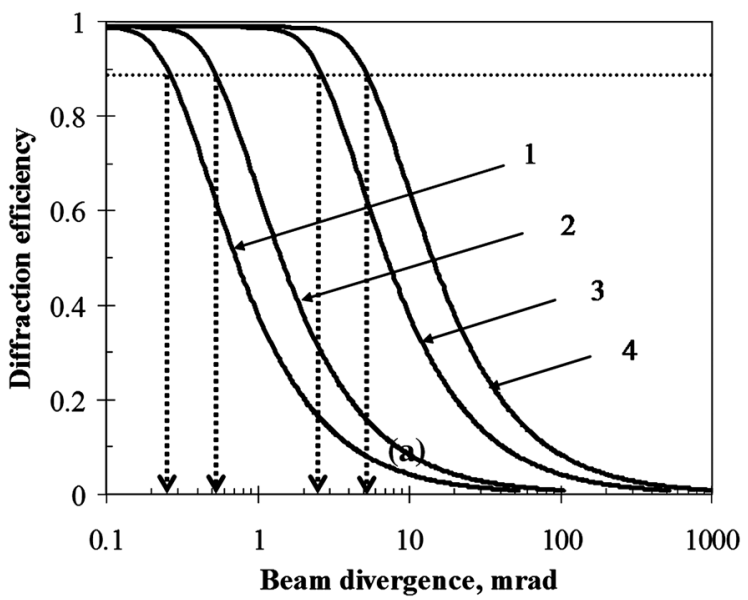

(c)

Fig. 9 Effect of divergence of incident beam on parametrs of 1.1-mmthick reflecting VBG. Diffraction efficiency of the grating for plane monochromatic wave is $99 \%$. (a) Dependence of diffraction efficiency on beam divergence for different incident Bragg angles in medium $\theta_{m}$, deg: $1=0 ; 2=0.5 ; 3=1.0 ; 4=5.0 ; 5=10.0$. HWFZ angular selectivity of grating for corresponding incident Bragg angles, mrad: $1=30.3,2=22.8,3=17.5,4=5.1,5=2.6$; shown by dotted arrows. (b) Dependence of angular selectivity (HWFZ) and diffraction efficiency on incident Bragg angle in a medium. Divergence of a beam is chosen equal to the grating selectivity at corresponding incident Bragg angles. (c) Dependence of diffraction efficiency on beam divergence for incident Bragg angle $\theta_{m}^{*}=10^{\circ}$. HWFZ angular selectivity of gratings, mrad: $1=0.26,2=0.53,3=2.6,4=5.2$; shown by dotted arrows. Grating thickness, $\mathrm{mm}: 1=10.8,2=5.4,3=1.08,4=0.54$. Dotted line corresponds to diffraction efficiency for a beam with the same beam divergence as the grating selectivity. 
requirement is much less restrictive for reflecting VBGs and is absolutely the same as was described in Sec. B.1 for interrelations between spectral parameters of the beam and reflecting grating. Generally, reflecting VBGs are less restrictive for achieving high $\mathrm{DE}$ in comparison with transmitting VBGs, especially for divergent beams, but they have narrower spectral selectivity which could considerably restrict efficient Bragg diffraction of wide-spectrum beams.

If considering diffraction of divergent polychromatic beams from reflecting VBGs, the total peak DE after the Bragg grating is a product of $\eta_{\lambda}(\Delta \lambda=0, w)$ and $\eta_{\theta}(\Delta \theta=$ $0, b)$ which is calculated from Eqs. (23) and (25):

$\eta(w, b)=\eta_{\lambda}(\Delta \lambda=0, w) \eta_{\theta}(\Delta \theta=0, b)$.

For instance, if a beam is characterized by both angular divergence and spectral bandwidth which is equal to the angular and spectral selectivity of a reflecting VBG, its diffraction efficiency will be about only $80 \%$ compared with $99 \%$ DE for the same grating in plane monochromatic wave.

In some cases it is desirable to calculate diffraction efficiency as a function of wavelength or angle while considering effects of a divergent beam and laser bandwidth simultaneously. In this case, simply multiplying Eqs. (23) and (25) will not be effective; instead, a double convolution must be performed. Equation (27) gives the resulting expression.

$$
\begin{aligned}
& \eta_{\theta}(\Delta \theta, b)=\frac{\left[\eta * G_{2}\right]\left(\Delta \lambda, \Delta \theta_{m}\right)}{\int G_{1}(\Delta \lambda, w) d \Delta \lambda \int G_{2}\left(\Delta \theta_{m}, b\right) d \Delta \theta_{m}} \\
& =\frac{\iint \eta\left(\lambda_{c}, \theta_{c}\right) G_{1}\left[\left(\Delta \lambda-\lambda_{c}\right), w\right] G_{2}\left[\left(\Delta \theta_{m}-\theta_{c}\right), b\right] d \theta_{c} d \lambda_{c}}{\int G_{1}(\Delta \lambda, w) d \Delta \lambda \int G_{2}\left(\Delta \theta_{m}, b\right) d \Delta \theta_{m}} .
\end{aligned}
$$

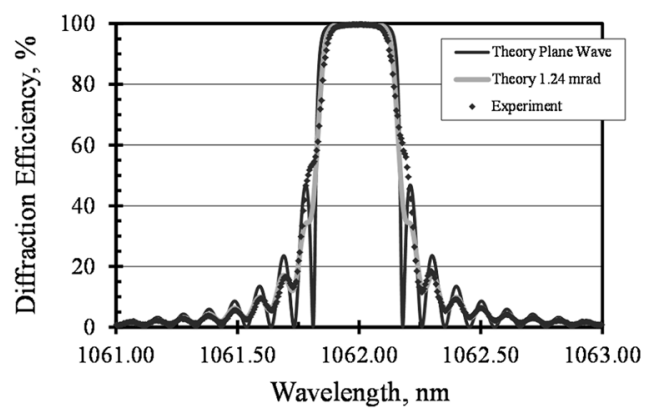

(a)

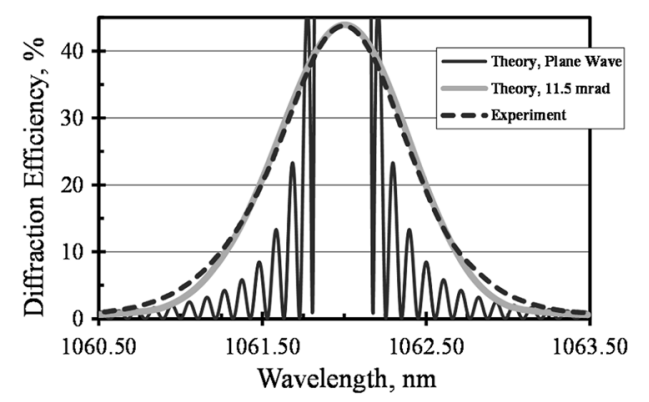

(b)

Fig. 10 Comparison of Modeling with experimental data. The VBG used is $3.5 \mathrm{~mm}$ thick with a refractive index modulation of $420 \mathrm{ppm}$, and has a spectral selectivity of $0.186 \mathrm{~nm}$ (HWFZ) and an angular selectivity of $3.67 \mathrm{mrad}$ (full angle at 5.4 deg Bragg angle in glass). (a) 1.24 mrad beam divergence; (b) 23 mrad beam divergence.

\section{Comparison of Modeling and Measurements}

When comparing experimental data with ideal VBGs modeled above, there are usually multiple contributors that deteriorate the diffraction efficiency. Effects of spectral width and angular divergence of laser beams have been discussed above. Effects of losses and nonuniformity of real VBGs requires additional modeling that will be a subject of future researches. However, it is clear that material losses would decrease diffraction efficiency while inhomogeneity in the VBG, which result in variations in Bragg wavelength and diffraction efficiency across the aperture, will contribute to the diffraction efficiency in much the same was as does spectral or angular divergence of laser beams.

Figure 10(a) shows the measured and modeled spectra of diffraction efficiency of a reflecting VBG at normal incidence. This grating is $3.5 \mathrm{~mm}$ thick and has a spectral selectivity HWFZ of $0.186 \mathrm{~nm}$ and angular selectivity of $3.67 \mathrm{mrad}$ (full-angle). The two curves match well when $1.24 \mathrm{mrad}$ beam divergence (full-angle) is assumed. To remove any ambiguity about which effect we are measuring, another experiment was performed in which the beam was focused onto a very small spot on the grating. In this way, we can reduce the effect from VBG inhomogeneity to be negligible. Figure 10(b) shows the measured and calculated data resulting from this experiment. The beam divergence was measured to be approximately $23 \mathrm{mrad}$ (full-angle), and calculations made by using this beam divergence match very well with the experimental data. In this high-divergence case, one can see that the side lobes have been completely washed out. This behavior was predicted above for cases in which the divergence, spectral or angular, exceeds the selectivity of the VBG. Spectrally divergent beams behave similarly.

\section{Conclusions}

Mathematical modeling based on Kogelnik's coupled wave theory has been produced for diffraction of beams with a wide range of spectral and angular parameters on reflecting volume Bragg gratings. This model is particularly important for most practical applications when spectral width and angular divergence of diffracted beams are comparable with spectral and angular selectivity of reflecting Bragg gratings. Contrary to fiber Bragg gratings described in numerous publications, free space VBGs allow for an additional design dimension where the interacting beams are not confined to a waveguide. This additional dimension allows for angular tuning of the Bragg resonance, and highly divergent (nonguided) beam interaction. The proposed model allows fast analytical calculation of angular and spectral dependencies of diffraction efficiency for normal and slanted gratings and could be used for the design of different devices based on reflecting Bragg gratings as well as testing tools for Bragg grating certification. This theoretical model was compared with and found very close to experimental data observed in Bragg mirrors recorded in photo-thermo-refractive (PTR) glass. This model can be applied for all other thick Bragg gratings with sinusoidal refractive index modulation recorded in different phase photosensitive materials. 


\section{Acknowledgments}

This work was supported by the DARPA/ADHELS program; contract number H0011-06-1-0010. The authors thank to Prof. Boris Zeldovich, Dr. Sergiy Mokhov, and Dr. Armen Sevian for fruitful discussions.

\section{References}

1. H. Kogelnik, "Coupled wave theory for thick hologram gratings," Bell Syst. Tech. J. 48(9), 2909-2945 (1969).

2. L. B. Glebov et al., "Reflection of light by composite volume holograms: Fresnel corrections and Fabry-Perot spectral filtering," JOSA A 25(3), 751-764 (2008).

3. I. V. Ciapurin, L. B. Glebov, and V. I. Smirnov, "Modeling of phase volume diffractive gratings, part 1: transmitting sinusoidal uniform gratings," Opt. Eng. 45(1), 015802 (2006).

4. M. G. Moharam, T. K. Gaylord, and R. Magnusson, "Bragg diffraction of finite beams by thick gratings," JOSA 70(3), 300-304 (1980).

5. J. Hellstrom and B. Jacobsson, "Finite beams in reflective volume Bragg gratings: theory and experiments," IEEE J. Quant. Electron. 44(1), 81-89 (2008).

6. R. Kashyap, "Theory of fiber Bragg gratings," Chapter 4 in Fiber Bragg Gratings, pp. 119-188, Academic Press, Burlington, MA (2010).

7. T. Erdogan, "Fiber grating spectra," J. Lightwave Tech. 15(8), 1277-1294 (1997).

8. A. W. Jones, J. Bland-Hawthorn, and P. L. Shopbell, "Towards a general definition for spectroscopic resolution," in Astronomical Data Analysis Software and Systems IV, Astronomy Society of the Pacific Conference Series, Vol. 77, pp. 503-506 (1995).

9. B. L. Volodin et al., "Wavelength stabilization and spectrum narrowing of high-power multimode laser diodes and arrays by use of volume Bragg gratings," Opt. Lett. 29(16), 1891-1893 (2004).

10. G. B. Venus et al., "Stable coherent coupling of laser diodes by a volume Bragg grating in photothermorefractive glass," Opt. Lett. 31(10), 1453-1455 (2006).

11. P. Jelger and F. Laurell, "Efficient narrow-linewidth volume-Bragg grating-locked Nd:fiber laser," Opt. Express 15(18), 11336-11340 (2007).

12. O. Andrusyak et al., "Spectral combining and coherent coupling of lasers by volume Bragg gratings," IEEE J. Sel. Top. Quant. 15(2), 344-353 (2009)

13. O.Andrusyak et al., "Fiber laser system with $\mathrm{kW}$-level spectrallycombined output," in 21st Solid State and Diode Laser Technology Review Proc., Directed Energy Professional Society, pp. 3-7 (2008).

14. A. Jain et al., "Coherent and spectral beam combining of fiber lasers using volume Bragg gratings," in Proc. SPIE 7686, 768615 (2010).

15. I. Divliansky et al., "Volume bragg lasers," Proc. SPIE 7751, 77510Z (2010).

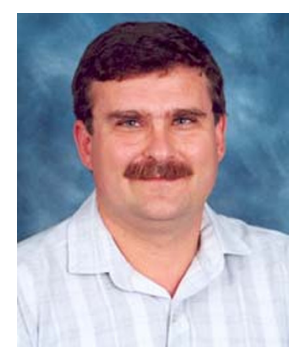

Igor V. Ciapurin earned his MS in optoelectronics from Kishinev State University, Moldova and his $\mathrm{PhD}$ in physics of semiconductors and dielectrics from the State University of Moldova. After receiving his $\mathrm{PhD}$ degree, he joined the State University of Moldova R\&D Department in 1991 and held various research and management positions. Since 2001, he has been with CREOL/UCF in Orlando, Florida, where his research has mainly aimed to design and develop volume Bragg gratings in PTR glass for spectral beam combining of highpower lasers. He has worked with OptiGrate Corporation since 2005. He has over 70 publications in refereed journals and conference proceedings, and over 15 years of experience in business management.

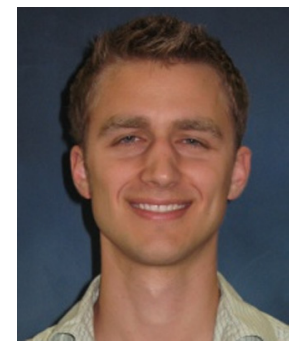

Derrek R. Drachenberg received his MS in optics in 2009, and his PhD in optics in 2011, from CREOL, the College of Optics and Photonics at the University of Central Florida in Orlando. In 2007, he joined the Photoinduced-processing lab at CREOL, where his research was focused on high-power spectral beam combining of fiber lasers. He is currently a Postdoctoral Research Staff Member at Lawrence Livermore National Labs in the NIF and Photon Sciences Directorate, where his work is in fiber lasers.

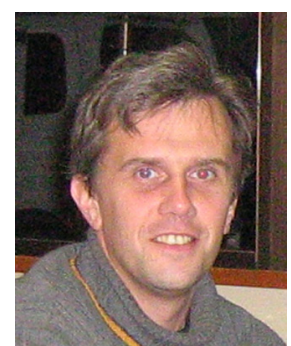

Vadim I. Smirnov is a co-founder and CTO of OptiGrate Corp. He is coauthor of more than 70 presentations and publications and five U.S. patents on high-efficiency diffractive elements in Photo-Thermo-Refractive glass. More than 15 years ago he joined Dr. Glebov's research group at School of Optics/ CREOL, where the technology PTR glass was developed. For the last 8 years he has led the development of holographic technology at OptiGrate Corp. His research activities include design and fabrication of volume Bragg gratings, their applications for laser engineering, spectroscopy, and high-power optics.

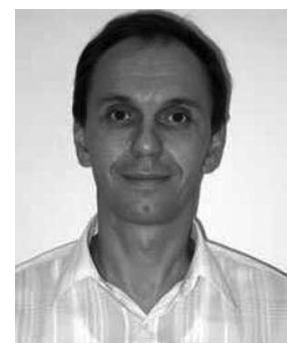

George B. Venus received his MS in electrical engineering from the Electro-Technical University, St. Petersburg, Russia, in 1984, and his PhD in physics from loffe PhysicoTechnical Institute, St. Petersburg, in 1991. From 1984 to 2003, he was a Research Scientist with loffe Institute. Since 2003, he has been a Research Scientist at the Center for Research and Education in Optics and Lasers (CREOL), The College of Optics and Photonics, University of Central Florida, Orlando. His current research interests include high-power semiconductor laser devices, external cavity semiconductor laser devices, and high-power multichannel laser systems.

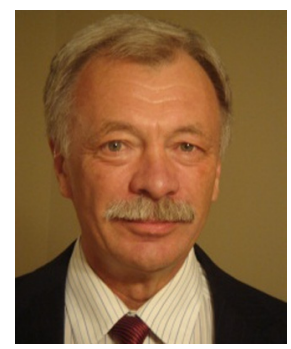

Leonid B. Glebov earned his $\mathrm{PhD}$ in physics (major in Optics) from State Optical Institute, Leningrad, Russia (1976). He was affiliated with that institute up to 1995. Since 1995 he has been at CREOL/The College of Optics and Photonics, University of Central Florida as a Research Professor. He has published a book and more than 300 journal papers; he also holds over 15 Russian patents, and 9 awarded and 3 pending U.S. patents. He is a fellow of the major optics and photonics societies, and a recipient of SPIE Denis Gabor award in holography. The main directions of his research are optical properties of glasses, holographic optical elements, and semiconductor, solid-state, and fiber lasers controlled by volume Bragg gratings. Leon Glebov is a founder and VP for R\&D of OptiGrate Corporation, which develops and fabricates holographic optical elements (volume Bragg gratings) for multiple applications in lasers and photonics. 